\title{
Acquired amegakaryocytic thrombocytopenic purpura induced by percutaneous ethanol injection during treatment of hepatocellular carcinoma: A case report
}

\author{
DING-LUN AI ${ }^{1 *}$, BO-TAO LI ${ }^{2 *}$, XIAO-MING PENG ${ }^{1}$, LIN-ZHI ZHANG $^{1}$, JING-YAN WANG $^{1}$, YUN ZHAO $^{1}$, \\ BIN YANG ${ }^{1}$, QIANG YU ${ }^{1}$, CHUN-ZI LIU ${ }^{1}$, NING YANG ${ }^{3}$, HUA-MING WANG $^{1}$ and LIN ZHOU ${ }^{1}$ \\ ${ }^{1}$ Department of Interventional Radiology, Beijing 302nd Hospital, Beijing 100039; \\ ${ }^{2}$ Department of Hematopoietic Stem Cell Transplantation, Affiliated Hospital to the Chinese Academy of \\ Military Medical Sciences, Beijing $100071 ;{ }^{3}$ Department of Clinical Laboratory, \\ Beijing 302nd Hospital, Beijing 100039, P.R. China
}

Received January 7, 2015; Accepted October 23, 2015

DOI: 10.3892/ol.2015.3934

\begin{abstract}
Percutaneous ethanol injection is an important localized treatment method for patients presenting with hepatocellular carcinoma (HCC). Among the advantages of percutaneous ethanol injection are its minimal invasiveness, simplicity, low cost and low risk of complications. However, the increasing popularity of percutaneous ethanol injection has resulted in serious adverse effects attributed to individual variations. The present study describes the case of a patient who exhibited acquired amegakaryocytic thrombocytopenic purpura, caused by percutaneous ethanol injection treatment for HCC. This complication was promptly identified, and platelet transfusion and injection of recombinant human interleukin-11 resulted in a rapid recovery of the patient's platelet count. Attention should be given to this rare complication in patients administered percutaneous ethanol injection treatment for HCC.
\end{abstract}

\section{Introduction}

Hepatocellular carcinoma (HCC) is a common malignancy and the third most common cause of cancer-associated mortality worldwide (1). The diagnosis of early-stage HCC is complex due to its non-specific symptoms and atypical features. The majority of patients are therefore diagnosed with late-stage disease or distant metastasis, with $<20 \%$ of patients eligible

Correspondence to: Dr Lin Zhou, Department of Interventional Radiology, Beijing 302nd Hospital, 100 Xisihuan Middle Road, Beijing 100039, P.R. China

E-mail: lindagery302@163.com

*Contributed equally

Key words: complication, hepatocellular carcinoma, percutaneous ethanol injection, acquired amegakaryocytic thrombocytopenic purpura for surgical treatment at the point of diagnosis (2). Over the previous 30 years, percutaneous ethanol injection (PEI) has become an important method for the treatment of patients exhibiting HCC. Among its advantages are its minimal invasiveness, simplicity, low cost and relatively low occurrence of complications $(3,4)$. However, PEI may give rise to serious adverse effects such as serious alcohol allergy, liver failure and abdominal bleeding (5). The present case report describes a patient exhibiting acquired amegakaryocytic thrombocytopenic purpura (AATP) caused by PEI with a reduction or complete absence of megakaryocytes in the bone marrow and resulting in reduced platelet counts in the peripheral blood.

\section{Case report}

A 49-year-old woman diagnosed with HCC and cirrhosis associated with hepatitis B virus (HBV) infection was admitted to Beijing 302nd Hospital on October 11, 2013. The patient's complete blood count test upon hospital admission showed the following: Leukocytes, 4.08 $\times 10^{9} / 1$ (normal range, $4 \times 10^{9} / 1$ ); erythrocytes, $2.84 \times 10^{12} / 1$ (normal range, $3.68-5.13 \times 10^{12} / 1$ ); hemoglobin, $106 \mathrm{~g} / \mathrm{l}$ (normal range, 113-151 g/l); and platelets, $56.00 \times 10^{9} / 1$ (normal range, $100-300 \times 10^{9} / 1$ ). The patient's coagulation function tests demonstrated a prothrombin time (PT)/prothrombin time activity (PTA) of $13.1 \mathrm{sec} / 79 \%$ (normal range, 11-14.3 sec/65-130\%). The patient was positive for hepatitis B surface antigen, with an HBV DNA quantity of $<40 \mathrm{U} / \mathrm{ml}$ (normal range, $<40 \mathrm{U} / \mathrm{ml}$ ). Liver function tests showed the following: Alanine aminotransferase (ALT), $34 \mathrm{U} / 1$ (normal range, 35-55 U/1); aspartate aminotransferase (AST), $23 \mathrm{U} / 1$ (normal range, 8-40 U/l); albumin, $35 \mathrm{~g} / \mathrm{l}$; total bilirubin (normal range, 35-55 g/l), $18 \mu \mathrm{mol} / 1$ (normal range, 3.4-20.5 $\mu \mathrm{mol} / \mathrm{l}$ ); and $\alpha$-fetoprotein, $1,210 \mathrm{ng} / \mathrm{ml}$ (normal range, 0-10 ng/ml). An electrocardiogram and a thoracic computed tomography scan did not reveal any abnormalities. Enhanced magnetic resonance imaging (Fig. 1) revealed a round focus, measuring $\sim 2.9 \times 2.1 \mathrm{~cm}$, in the anterior portion of the right lobe of the liver. The focus was significantly enhanced in the arterial phase, while the contrast agent faded in the portal and 


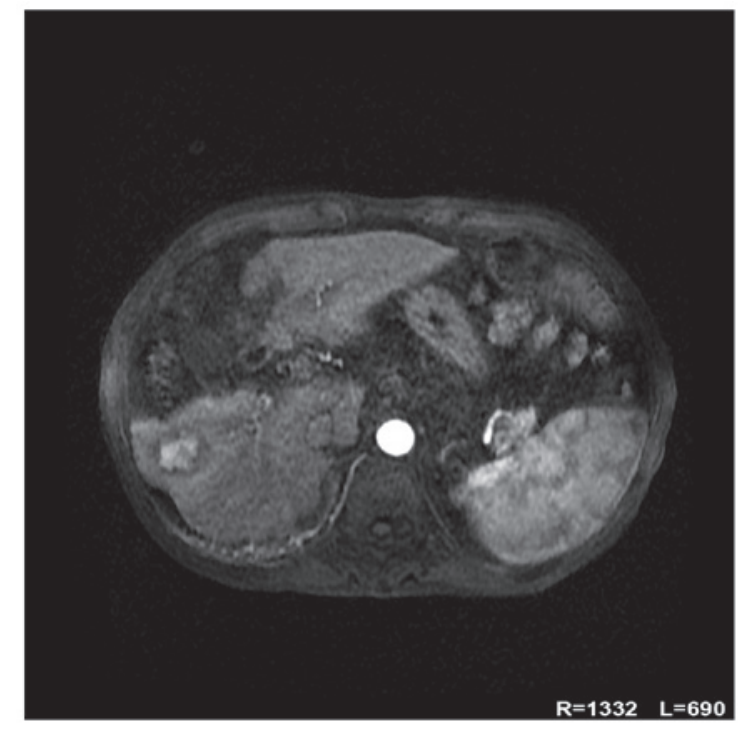

Figure 1. Enhanced magnetic resonance imaging revealing one tumor nodule in the anterior portion of the right lobe of the liver, measuring $\sim 2.9 \times 2.1 \mathrm{~cm}$.

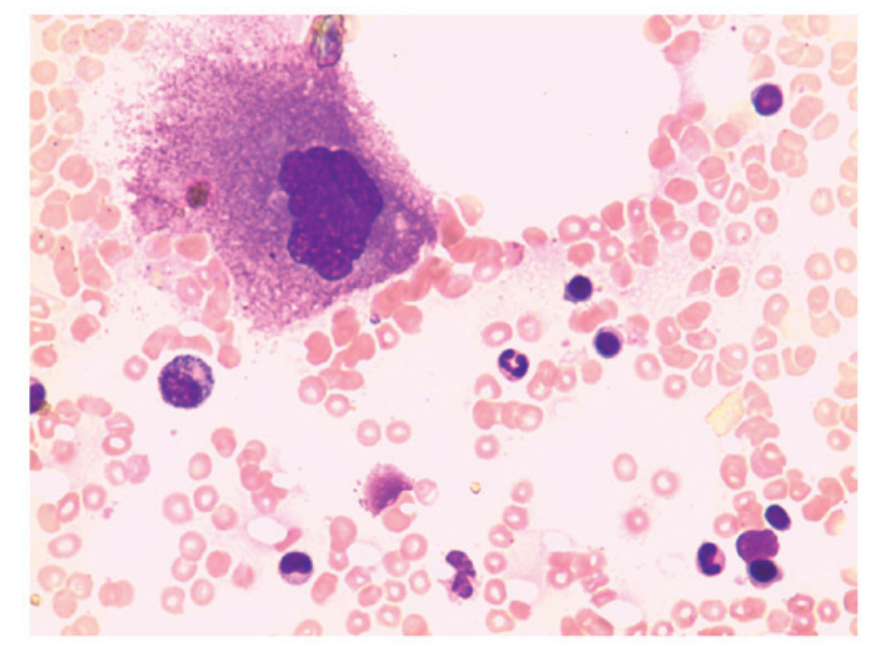

Figure 2. Bone marrow analysis revealing active proliferation of granulocytes and erythrocytes with normal morphology. Megakaryocytes, however, showed a marked decrease in number and no mature megakaryocytes were observed.

delayed phases. These findings were consistent with a diagnosis of HCC.

The patient refused surgical resection and transarterial chemoembolization (TACE). Radiofrequency ablation (RFA) was not a suitable treatment as the tumor nodule was adjacent to the intestinal canal. The patient was treated with PEI, with the focus receiving a total dose of $5 \mathrm{ml}$ anhydrous ethanol. The morning subsequent to treatment, the patient discovered multiple petechiae on all four limbs. The patient did not experience gum bleeding, hemoptysis, hematemesis, melena, palpitations, chest discomfort, abdominal pain or cervical rigidity. An emergency complete blood count showed the following: Leukocytes,

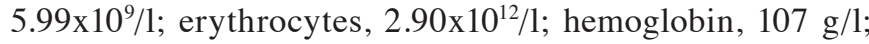
and platelets, $2.00 \times 10^{9} / 1$. Coagulation function tests revealed a PT/PTA of $13.1 \mathrm{sec} / 77.6 \%$. Liver function tests indicated the following: ALT, $45 \mathrm{U} / \mathrm{l}$; AST, $56 \mathrm{U} / \mathrm{l}$; albumin, $36 \mathrm{~g} / \mathrm{l}$; and total bilirubin, $22 \mu \mathrm{mol} / \mathrm{l}$. Upon platelet transfusion and injection of recombinant human interleukin (IL)-11, the patient's platelet count rose to $30.00 \times 10^{9} / 1$. Two bone marrow punctures were performed to confirm the diagnosis. A cytological study (Fig. 2) revealed active proliferation of granulocytes and erythrocytes. These granulocytes and erythrocytes, as well as lymphoid cells, demonstrated a typical morphology. Megakaryocytes, however, showed a marked decrease in number and a maturation defect. The patient was diagnosed with AATP, and received a second platelet transfusion and injection of recombinant human IL-11, which increased the platelet count to $50.00 \times 10^{9} / 1$. A physical examination revealed the disappearance of petechiae from all four limbs. A complete blood count 3 months after treatment showed the following: Leukocytes, $4.66 \times 10^{9} / 1$; erythrocytes, $3.13 \times 10^{12} / 1$; haemoglobin, $113 \mathrm{~g} / 1$; and platelets, $34.00 \times 10^{9} / 1$.

The patient was discharged when all the symptoms had disappeared, and regular reviews were undertaken in the local hospital. The regular follow-up phone calls revealed that the patient remained stable.

\section{Discussion}

Treatment options for patients exhibiting inoperable HCC include TACE, RFA, argon-helium cryoablation and PEI. PEI has been utilized to treat HCC lesions $<3 \mathrm{~cm}$ in diameter (6). The 1-, 3- and 5-year overall survival rates in patients treated with PEI for HCCs $<5 \mathrm{~cm}$ in diameter have been reported as 95, 75 and 59\%, respectively (7). The most common complications of PEI treatment for HCC are abdominal pain and a self-limiting fever (8). In a study of 746 patients with HCC who underwent PEI, only 13 (1.7\%) exhibited serious adverse reactions, including hemorrhage in 5 patients, pleuritis in 2 , hemobilia in 2 and a hepatic abscess in 1 patient (9). Similarly, in a study of $2,147 \mathrm{HCC}$ patients who received PEI, only $45(2.1 \%)$ experienced serious adverse reactions (4). Serious hypotension has additionally been reported in two patients following PEI treatment (10). Thus, compared with alternative treatment regimens, PEI treatment is effective, cheap and safe, leading to fewer adverse effects in patients with HCC.

AATP is a clinically rare bleeding disease, characterized by a decrease or complete absence of megakaryocytes in the bone marrow and resulting in decreased platelet counts in the peripheral blood (11). By contrast, the remaining hematopoietic lineages are normal $(12,13)$. AATP is not associated with patient age, however, it is more common in women, particularly young women, compared with men, whilst being rarely observed in children. The typical clinical presentation of AATP is a marked decrease in platelet count in the peripheral blood (14). The etiology of AATP remains to be elucidated, and it may be associated with drugs, infections, autoimmune diseases and radiation exposure $(12,13)$. Certain drugs have been observed to selectively inhibit megakaryocytes, including thiazide diuretics, estrogen, acetaminophen, alcohol and others $(13,15)$.

In certain alcoholic patients, thrombocytopenia is primarily a result of direct marrow suppression of platelet production following alcohol consumption $(16,17)$. Suppression of platelet production that is sufficient enough to produce thrombocytopenia requires consumption of large quantities of ethanol over several days (18). However, a study in which guinea pigs were allowed to ingest ethanol ad libitum showed that, although blood ethanol did not reach measurable levels, 
the average platelet count declined by $16 \%$ during the 4 -week study period (19). Thrombocytopenia induced by alcohol ingestion is accompanied by a decrease in the number of marrow megakaryocytes. Vacuolated proerythroblasts and granulocyte precursors may be observed, as well as multinuclear erythroblasts and megaloblasts. Vacuolization of the periphery of mature megakaryocytes has been observed (17). Thrombocytopenia typically resolves in 5-21 days following cessation of ethanol ingestion, occasionally with transient rebound thrombocytosis (16).

Prior to the onset of thrombocytopenia, the current patient received PEI, which was the probable cause of the AATP. Thus, we speculate that this adverse reaction may be due to the selective inhibition of megakaryocyte maturation by ethanol, leading to thrombocytopenia. Multiple blood tests for the patient demonstrated a significant reduction in platelet count, excluding the possibility of pseudothrombocytopenia. Cytological examination of the patient's bone marrow suggested normal or hyperproliferation of all hematopoietic lineages, with the exception of megakaryocytes, in which a defect in maturation was observed. Following the diagnosis of AATP, the patient received platelet transfusions and injections of recombinant human IL-11. The platelet count rose to $50.00 \times 10^{9} / 1$, and the patient was subsequently discharged.

IL-11 was approved by the US Food and Drug Administration for the treatment of bone marrow failure diseases and thrombocytopenia following chemotherapy in November 1997 (20). IL-11 augments the growth of megakaryocytic progenitors in the presence of IL-3, and acts to promote megakaryocyte maturation rather than proliferation (16). IL-11 is a multifunctional regulator of hematopoiesis in bone marrow stromal cells, and has a significant regulatory role in bone marrow megakaryocytes $(21,22)$. Due to the megakaryocyte maturation inhibition observed in the current patient, IL-11 treatment was administered and exerted a significant effect.

Although PEI for the treatment of HCC is relatively safe and effective, with few adverse effects, complications may occur due to individual variations. To manage such cases as the present case of post-PEI AATP, we recommend the routine monitoring of the platelet count of all patients undergoing PEI, which will enable the early discovery and timely management of this complication.

\section{References}

1. Jemal A, Bray F, Center MM, Ferlay J, Ward E and Forman D: Global cancer statistics. CA Cancer J Clin 61: 69-90, 2011.

2. Said A and Wells J: Management of hepatocellular carcinoma. Minerva Med 100: 51-68, 2009.

3. Livraghi T, Benedini V, Lazzaroni S, Meloni F, Torzilli G and Vettori C: Long term results of single session percutaneous ethanol injection in patients with large hepatocellular carcinoma. Cancer 83: 48-57, 1998.

4. Shiina S, Tateishi R, Imamura M, Teratani T, Koike Y, Sato S, Obi S, Kanai F, Kato N, Yoshida H, et al: Percutaneous ethanol injection for hepatocellular carcinoma: 20-year outcome and prognostic factors. Liver Int 32: 1434-1442, 2012.
5. Weis S, Franke A, Berg T, Mössner J, Fleig WE and Schoppmeyer K:Percutaneous ethanol injection or percutaneous acetic acid injection for early hepatocellular carcinoma.Cochrane Database Syst Rev 1: CD006745, 2015.

6. Ebara M, Kita K, Yoshikawa M and Ohto M: Non-vascular interventional radiology - percutaneous ethanol injection (PEI) in hepatocellular carcinoma smaller than $3 \mathrm{~cm}$ in diameter. Gan To Kagaku Ryoho 16: 3311-3318, 1989 (In Japanese).

7. Morimoto M, Numata K, Sugimori K, Shirato K, Kokawa A, Oka H, Hirasawa K, Koh R, Nihommatsu H and Tanaka K: Successful initial ablation therapy contributes to survival in patients with hepatocellular carcinoma. World J Gastroenterol 13: 1003-1009, 2007

8. Castroagudín JF, Delgado M, Villanueva A, Bustamante M, Martínez J, Otero E, Tomé S, Martínez SM, Segade FR, Conde R, et al: Safety of percutaneous ethanol injection as neoadjuvant therapy for hepatocellular carcinoma in waiting list liver transplant candidates. Transplant Proc 37: 3871-3873, 2005.

9. Livraghi T, Giorgio A, Marin G, Salmi A, de Sio I, Bolondi L, Pompili M, BrunelloF, Lazzaroni S and Torzilli G: Hepatocellular carcinoma and cirrhosis in 746 patients: Long-term results of percutaneous ethanol injection. Radiology 197: 101-108, 1995.

10. Gelczer RK, Charboneau JW, Hussain S and Brown DL: Complications of percutaneous ethanol ablation. J Ultrasound Med 17: 531-533, 1998.

11. Lai DW, Loughran TP Jr, Maciejewski JP, Sasu S, Song SX, Epling-Burnette PK and Paquette RL: Acquired amegakaryocytic thrombocytopenia and pure red cell aplasia associated with an occult large granular lymphocyte leukemia. Leuk Res 32: 823-827, 2008.

12. Quintás-Cardama A: Acquired amegakaryocytic thrombocytopenic purpura successfully treated with limited cyclosporin A therapy. Eur J Haematol 69: 185-186, 2002.

13. Tristano AG: Acquired amegakaryocytic thrombocytopenic purpura: Review of a not very well-defined disorder. Eur J Intern Med 16: 477-481, 2005.

14. Hirsh EH, Vogler WR, McDonald TP and Stein SF: Acquired hypomegakaryocytic thrombocytopenic purpura. Occurrence in a patient with absent thrombopoietic stimulating factor. Arch Intern Med 140: 721-723, 1980.

15. Gewirtz AM and Hoffman R: Transitory hypomegakaryocytic thrombocytopenia: Aetiological association with ethanol abuse and implications regarding regulation of human megakaryocytopoiesis. Br J Haematol 62: 333-344, 1986.

16. Kaushansky K, Lichtman MA, Beutler E, Kipps TJ, Seligsohn U and Prchal J (eds): Williams Hematology. 8th edition. McGraw-Hill, New York, NY, 2010.

17. Latvala J, Parkkila S and Niemelä O: Excess alcohol consumption is common in patients with cytopenia: Studies in blood and bone marrow cells. Alcohol Clin Exp Res 28: 619-624, 2004.

18. Tristano AG: Acquired amegakaryocytic thrombocytopenic purpura: Review of a not very well-defined disorder. Eur J Int Med 16: 477-481, 2005.

19. Smith CM 2nd, Tobin JD Jr, Burris SM and White JG: Alcohol consumption in the guinea pig is associated with reduced megakaryocyte deformability and platelet size. J Lab Clin Med 120: 699-706, 1992.

20. Kantarjian H, Giles F, List A, Lyons R, Sekeres MA, Pierce S, Deuson R and Leveque J: The incidence and impact of thrombocytopenia in myelodysplastic syndromes. Cancer 109: 1705-1714, 2007.

21. Weich NS, Wang A, Fitzgerald M, Neben TY, Donaldson D, Giannotti J, Yetz-Aldape J, Leven RM and Turner KJ: Recombinant human interleukin-11 directly promotes megakaryocytopoiesis in vitro. Blood 90: 3893-3902, 1997.

22. Cantor SB, Elting LS, Hudson DV Jr and Rubenstein EB: Pharmacoeconomic analysis of oprelvekin (recombinant human interleukin-11) for secondary prophylaxis of thrombocytopenia in solid tumor patients receiving chemotherapy. Cancer 97: 3099-3106, 2003. 